\title{
NONLINEAR OSCILLATION OF A SUBLINEAR DELAY EQUATION OF ARBITRARY ORDER
}

\author{
TAKAŜSI KUSANO AND HIROSHI ONOSE
}

Abstract. The equations considered generalize

$$
x^{(n)}(t)+p(t)|x(g(t))|^{\alpha} \operatorname{sgn} x(g(t))=0, \quad 0<\alpha<1 .
$$

A necessary and sufficient condition is established that all solutions are oscillatory when $n$ is even and are either oscillatory or strongly monotone when $n$ is odd. The result makes clear a difference in oscillatory property between sublinear delay equations and the corresponding ordinary differential equations.

We consider the nonlinear delay equation

$$
x^{(n)}(t)+p(t) f(t, x(t), x(g(t)))=0,
$$

where the following conditions are always assumed to hold:

(a) $p(t)$ is continuous and nonnegative on $R_{+}=[0, \infty)$;

(b) $g(t)$ is continuous on $R_{+}$and such that $g(t) \leqq t, \lim _{t \rightarrow \infty} g(t)=\infty$;

(c) $f(t, x, y)$ is continuous on $S=R_{+} \times R \times R$ and such that $y f(t, x, y)>$ 0 for $(t, x, y) \in S$ with $y \neq 0$.

We tacitly assume that under the initial condition

$$
x(t)=\phi(t), \quad t \leqq t_{0} \quad \text { and } \quad x^{(j)}\left(t_{0}\right)=x_{j}^{0}, \quad j=1, \cdots, n-1,
$$

equation (1) has a solution which can be continued to $\left[t_{0}, \infty\right)$.

A nontrivial solution $x(t)$ of (1) is called oscillatory if there exists a sequence $\left\{t_{k}\right\}_{k=1}^{\infty}$ such that $x\left(t_{k}\right)=0$ for all $k$ and $\lim _{k \rightarrow \infty} t_{k}=\infty$. Otherwise, a solution is called nonoscillatory. A nonoscillatory solution is said to be strongly monotone if it tends monotonically to zero as $t \rightarrow \infty$ together with its first $n-1$ derivatives.

The object of this paper is to establish under appropriate restrictions on $f$ a necessary and sufficient condition that every solution of (1) be oscillatory in the case $n$ is even and be either oscillatory or strongly monotone in the case $n$ is odd. Our theorem generalizes to arbitrary $n \geqq 2$ those of Gollwitzer [2] and Ševelo and Odarič [10] for the second order delay

Received by the editors October 5, 1972 and, in revised form, December 26, 1972.

AMS (MOS) subject classifications (1970). Primary 34C10, 34K15.

Key words and phrases. Oscillatory, nonoscillatory, sublinear, nonlinear delay equation.

(c) American Mathematical Society 1973 
equation $x^{\prime \prime}(t)+p(t)[x(g(t))]^{\alpha}=0$, where $\alpha$ is the ratio of odd positive numbers and $\alpha<1$. (We note that Gollwitzer's theorem has been extended to a class of second order functional differential equations by Burkowski [1].) Our results show how the rate of growth for large $t$ of the retarded argument $g(t)$ affects the oscillatory property of delay equations in question.

THEOREM 1. Suppose there exist positive constants $K$ and $\alpha<1$ such that

$$
|f(t, x, y)| \leqq K|y|^{\alpha} \quad \text { for }(t, x, y) \in S .
$$

Then a necessary condition that every solution of (1) be oscillatory if $n$ is even and be either oscillatory or strongly monotone if $n$ is odd is that

$$
\int^{\infty}[g(t)]^{\alpha(n-1)} p(t) d t=\infty .
$$

Proof. The proof is based on the arguments developed by Waltman [13], Hallam [3], Singh [12] and Ladas [7].

We assume that (3) does not hold and demonstrate that equation (1) has a nonoscillatory solution $x(t)$ such that $\lim _{t \rightarrow \infty} x(t) / t^{n-1}=a \neq 0$.

Choose $t_{0}$ so large that $g(t)>0$ for $t \geqq t_{0} \geqq 1$ and integrate (1) $n$ times from $t_{0}$ to $t$. Then we have

$$
x(t)=\sum_{j=0}^{n-1} \frac{x^{(j)}\left(t_{0}\right)}{j !}\left(t-t_{0}\right)^{j}-\frac{1}{(n-1) !} \int_{t_{0}}^{t}(t-s)^{n-1} p(s) f(s, x(s), x(g(s))) d s,
$$

which yields, in view of (2),

$$
|x(t)| \leqq C t^{n-1}+K t^{n-1} \int_{t_{0}}^{t} p(s)|x(g(s))|^{\alpha} d s, \quad t \geqq t_{0},
$$

where $C$ is a positive constant.

We define the function $F(t)$ by

Then

$$
F(t)=C+K \int_{t_{0}}^{t} p(s)|x(g(s))|^{\alpha} d s .
$$

$$
|x(t)| \leqq t^{n-1} F(t), \quad t \geqq t_{0} .
$$

If we choose $t_{1} \geqq t_{0}$ so large that $g(t) \geqq t_{0}$ for $t \geqq t_{1}$, it follows from (6) and the increasing character of $F(t)$ that

$$
|x(g(t))| \leqq[g(t)]^{n-1} F(g(t)) \leqq[g(t)]^{n-1} F(t), \quad t \geqq t_{1} .
$$

From (5) and (7) we have

$$
F^{\prime}(t)=K p(t)|x(g(t))|^{\alpha} \leqq K p(t)[g(t)]^{\alpha(n-1)} F(t)^{\alpha}, \quad t \geqq t_{1},
$$


and consequently,

$$
\begin{aligned}
F(t) & \leqq\left\{F\left(t_{1}\right)^{1-\alpha}+(1-\alpha) K \int_{t_{1}}^{t}[g(s)]^{\alpha(n-1)} p(s) d s\right\}^{1 /(1-\alpha)} \\
& \leqq\left\{F\left(t_{1}\right)^{1-\alpha}+(1-\alpha) K \int_{t_{1}}^{\infty}[g(s)]^{\alpha(n-1)} p(s) d s\right\}^{1 /(1-\alpha)} \equiv C_{1},
\end{aligned}
$$

where $C_{1}$ is a finite positive constant. The inequalities (6) and (7) then become

$$
\begin{gathered}
|x(t)| \leqq C_{1} t^{n-1}, \quad t \geqq t_{1}, \\
|x(g(t))| \leqq C_{1}[g(t)]^{n-1}, \quad t \geqq t_{1} .
\end{gathered}
$$

Now we integrate (1) from $t_{1}$ to $t$ to obtain

$$
x^{(n-1)}(t)=x^{(n-1)}\left(t_{1}\right)-\int_{t_{1}}^{t} p(s) f(s, x(s), x(g(s))) d s,
$$

from which and in view of (2), (3), $\left(7^{\prime}\right)$ we conclude that the finite limit $\lim _{t \rightarrow \infty} x^{(n-1)}(t)$ exists. If we require that

$$
x^{(n-1)}\left(t_{1}\right)>C_{1} K \int_{t_{1}}^{\infty}[g(s)]^{\alpha(n-1)} p(s) d s,
$$

then this limit of $x^{(n-1)}(t)$ is not zero, and the solution $x(t)$ has the desired asymptotic property.

THEOREM 2. Suppose there exist positive constants $k$ and $\beta<1$ such that

$$
|f(t, x, y)| \geqq k|y|^{\beta} \quad \text { for }(t, x, y) \in S \text {. }
$$

If

$$
\int^{\infty}[g(t)]^{\beta(n-1)} p(t) d t=\infty,
$$

then every solution of (1) is oscillatory in the case $n$ is even and is either oscillatory or strongly monotone in the case $n$ is odd.

The following lemma of Kiguradze [4] will be needed.

LEMMA. If $x(t), x^{\prime}(t), \cdots, x^{(n-1)}(t)$ are absolutely continuous and of constant sign on the interval $\left[t_{0}, \infty\right)$, and $x^{(n)}(t) x(t) \leqq 0$, then there exists an integer $l, 0 \leqq l \leqq n-1$, which is even if $n$ is odd and odd if $n$ is even, such that

$$
|x(t)| \geqq \frac{\left(t-t_{0}\right)^{n-1}}{(n-1) \cdots(n-l)}\left|x^{(n-1)}\left(2^{n-l-1} t\right)\right|, \quad t \geqq t_{0} .
$$


Proof of Theorem 2. Our proof is an adaptation of the arguments developed by Ryder and Wend [9] for the case $g(t) \equiv t$ and is similar to that used by Ševelo and Vareh [11] for even order linear delay equations.

Let $n$ be even and let $x(t)$ be a nonoscillatory solution of (1). We may assume that $x(t)>0$ for large $t$. From the fact that $x^{(n)}(t)<0$ for large $t$, it follows that $x^{(n-1)}(t)$ is decreasing and that the derivatives of $x(t)$ of orders up to $n-1$ are eventually of constant sign, the odd order derivatives being eventually positive. In particular, $x^{\prime}(t)>0$, so that $x(t)$ is increasing for large $t$. According to Kiguradze's lemma we have

$$
x(t) \geqq x\left(2^{l-n+1} t\right) \geqq \frac{2^{(l-n+1)(n-1)}\left(t-t_{0}\right)^{n-1}}{(n-1) \cdots(n-l)} x^{(n-1)}(t)
$$

for $t \geqq t_{0}$, provided $t_{0}$ is sufficiently large. Therefore,

$$
x(t) \geqq A t^{n-1} x^{(n-1)}(t), \quad t \geqq t_{1}=2 t_{0},
$$

where $A=2^{(l-n+1)(n-1)} /(n-1) \cdots(n-l)$. Since $\lim _{t \rightarrow \infty} g(t)=\infty$, there is a $t_{2} \geqq t_{1}$ such that $g(t) \geqq t_{1}$ for $t \geqq t_{2}$. From (10) and the decreasing character of $x^{(n-1)}(t)$ we then have

$$
x(g(t)) \geqq A[g(t)]^{(n-1)} x^{(n-1)}(t), \quad t \geqq t_{2} .
$$

Combining (1) with (8) and (11) gives

$$
x^{(n)}(t)+A^{\beta} p(t)[g(t)]^{\beta(n-1)}\left[x^{(n-1)}(t)\right]^{\beta} \leqq 0 .
$$

Dividing by $\left[x^{(n-1)}(t)\right]^{\beta}$ and integrating form $t_{2}$ to $t$ we obtain

$$
\left.\frac{\left[x^{(n-1)}(s)\right]^{1-\beta}}{1-\beta}\right|_{t_{2}} ^{t}+A^{\beta} \int_{t_{2}}^{t}[g(t)]^{\beta(n-1)} p(s) d s \leqq 0,
$$

which implies $\int_{t_{2}}^{\infty}[g(t)]^{\beta(n-1)} p(t) d t<\infty$, a contradiction.

The case where $x(t)<0$ for large $t$ can be treated similarly.

Let $n$ be odd and assume the existence of a nonoscillatory solution $x(t)$. If $x(t)$ does not approach zero as $t \rightarrow \infty$, then, writing

$$
|x(t)|=\left|x(t) / x\left(2^{l-n+1} t\right)\right| \cdot\left|x\left(2^{l-n+1} t\right)\right|,
$$

applying Kiguradze's formula to $\left|x\left(2^{l-n+1} t\right)\right|$ and using the decreasing character of $\left|x^{(n-1)}(t)\right|$, we have

and

$$
|x(t)| \geqq M A t^{n-1}\left|x^{(n-1)}(t)\right|, \quad t \geqq t_{1},
$$

$$
|x(g(t))| \geqq M A[g(t)]^{n-1}\left|x^{(n-1)}(t)\right|, \quad t \geqq t_{2}
$$

where $M=\inf _{t \geqq t_{0}}|x(t)| x\left(2^{l-n+1} t\right) \mid$. The proof now proceeds exactly as in the case of even $n$. Thus it follows that a nonoscillatory solution of (1), if it 
exists, must approach zero as $t \rightarrow \infty$. In this case, not only $x(t)$ but also its first $n-1$ derivatives tend monotonically to zero as $t \rightarrow \infty$.

REMARK. Under some additional smoothness assumptions on $g(t)$, oscillation criteria of the form (9) were obtained by Ševelo and Odarič [10] for second order equations and by the present authors ([5], [6]) for higher order equations.

Combining Theorems 1 and 2 we obtain the following

THEOREM 3. Suppose there exist positive constants $k, K, \alpha<1$ such that

$$
k|y|^{\alpha} \leqq|f(t, x, y)| \leqq K|y|^{\alpha} \quad \text { for }(t, x, y) \in S .
$$

Then a necessary and sufficient condition that every solution of (1) be oscillatory when $n$ is even and be either oscillatory or strongly monotone when $n$ is odd is that (3) be valid.

REMARK. If $g(t)$ is of the form $g(t)=t-\tau(t)$ with $0 \leqq \tau(t) \leqq M$, then (3) is equivalent to

$$
\int^{\infty} t^{\alpha(n-1)} p(t) d t=\infty .
$$

Thus Theorem 3 is an extension of a theorem of Gollwitzer [2, Theorem 2] for the second order sublinear delay equation.

REMARK. On the basis of Theorem 3 we can compare the oscillatory property of sublinear delay equations with that of the corresponding differential equations without delay. As an illustration we consider

$$
x^{(n)}(t)+p(t)|x(t)|^{\alpha} \operatorname{sgn} x(t)=0
$$

and

$$
x^{(n)}(t)+p(t)|x(g(t))|^{\alpha} \operatorname{sgn} x(g(t))=0,
$$

where $n$ is even and $0<\alpha<1$. It is well known ([4], [8]) that all solutions of (13) are oscillatory if and only if (12) holds. Therefore, if $g(t)$ is such that the integrals in (3) and (12) converge or diverge simultaneously, e.g., if $\lim _{t \rightarrow \infty} g(t) / t=c>0$, then equations (13) and (14) have the same oscillatory property. It may happen that (12) holds but (3) does not. In this case, all solutions of (13) are oscillatory, while among solutions of (14) there is a nonoscillatory solution.

ACKNOWLEDGMENT. The authors wish to thank the referee for his helpful suggestions.

\section{REFERENCES}

1. F. Burkowski, Nonlinear oscillation of a second order sublinear functional differential equation, SIAM J. Appl. Math. 21 (1971), 486-490.

2. H. E. Gollwitzer, On nonlinear oscillation for a second order delay equation, J. Math. Anal. Appl. 26 (1969), 385-389. MR 39 \#581. 
3. T. G. Hallam, Asymptotic behavior of the solutions of an nth order nonhomogeneous ordinary differential equation, Trans. Amer. Math. Soc. 122 (1966), 177-194. MR 32 \#6000.

4. I. T. Kiguradze, On the question of variability of solutions of nonlinear differential equations. Differencial'nye Uravnenija 1 (1965), 995-1006=Differential Equations 1 (1965), 773-782. MR 33 \#2896.

5. T. Kusano and $\mathrm{H}$. Onose, Oscillation of solutions of nonlinear differential delay equations of arbitrary order, Hiroshima Math. J. 2 (1972), 1-13.

6. - Oscillation theorems for delay equations of arbitrary order, Hiroshima Math. J. 2 (1972), 263-270.

7. G. Ladas, Oscillation and asymptotic behavior of solutions of differential equations with retarded argument, J. Differential Equations 10 (1971), 281-290.

8. I. Ličko and M. Švec, Le caractère oscillatoire des solutions de l'équation $y^{(n)}+$ $f(x) y^{\alpha}=0, n>1$, Czechoslovak Math. J. 13 (88) (1963), 481-491. MR 28 \#4210.

9. G. H. Ryder and D. V. V. Wend, Oscillation of solutions of certain ordinary differential equations of nth order, Proc. Amer. Math. Soc. 25 (1970), 463-469. MR 41 \#5710.

10. V. N. Ševelo and O. N. Odarič, Certain questions of the theory of oscillation (nonoscillation) of solutions of second order differential equations with retarded argument, Ukrain. Mat. Ž. 23 (1971), 508-516. (Russian) MR 44 \#7088.

11. V. N. Ševelo and N. V. Vareh, On oscillability of solutions of higher order linear differential equations with retarded argument, Ukrain. Mat. Ž. 24 (1972), 513-520. (Russian)

12. Y.P. Singh, The asymptotic behavior of solutions of a nonlinear $n$ th-order differential equation, Okayama Math. J. 15 (1971), 71-73.

13. P. Waltman, On the asymptotic behavior of solutions of an nth order equation, Monatsh. Math. 69 (1965), 427-430. MR 32 \#2686.

Department of Mathematics, Hiroshima University, Hiroshima, Japan

Department of Mathematics, Ibaraki University, Mito, Japan 